\title{
Secular determinants of random unitary matrices
}

\author{
Fritz Haake†, Marek Kuśł $\dagger$, Hans-Jürgen Sommers $\dagger$, Henning Schomerus $\dagger$ \\ and Karol Życzkowski†£ \\ $\dagger$ Fachbereich Physik, Universität GH Essen, 45117 Essen, Germany \\ $\ddagger$ Center for Theoretical Physics and College of Science, Polish Academy of Sciences, PL-02-668 \\ Warszawa, Poland \\ $\S$ Instytut Fizyki im. Mariana Smoluchowskiego, Uniwersytet Jagielloński, ul Reymonta 4, \\ PL-30-059 Kraków, Poland \\ Received 19 January 1996
}

\begin{abstract}
We consider the characteristic polynomials of random unitary matrices $U$ drawn from various circular ensembles. In particular, the statistics of the coefficients of these polynomials are studied. The variances of these 'secular coefficients' are given explicitly for arbitrary dimension and continued analytically to arbitrary values of the level repulsion exponent $\beta$. The latter secular coefficients are related to the traces of powers of $U$ by Newton's well known formulae. While the traces tend to have Gaussian distributions and to be statistically independent among one another in the limit as the matrix dimension grows large, the secular coefficients exhibit strong mutual correlations due to Newton's mixing of traces to coefficients. These results might become relevant for current efforts at combining semiclassics and random-matrix theory in quantum treatments of classically chaotic dynamics.
\end{abstract}

\section{Introduction}

Circular ensembles of unitary matrices were first considered by Dyson [1] and described in detail by Mehta [2]. They are used to describe the quantum statistics of periodically driven systems (see [3] and references therein) and of scattering processes [4]. It is the unitary Floquet operator in the first case and the unitary $S$-matrix in the second that the random unitary matrices in question attempt to mimic with respect to certain more or less universal properties. Systems with global chaos in their classical limit and with time reversal invariance either present or strongly broken exhibit the greatest degree of universality in their statistical properties and tend to fall into one of the universality classes represented by the circular orthogonal, symplectic, or unitary ensembles (COE, CSE, CUE). The socalled Poissonian ensemble (CPE) of diagonal unitary matrices with independent unimodular eigenvalues has also found applications for certain classically integrable systems. There has been a recent interest in the analysis of intermediate ensembles of unitary matrices which describe the crossover between different universality classes [5-7]. Direct links between corresponding Gaussian and circular ensembles have been established lately [8,9].

Unitarity constraints imposed on a random matrix of small size $N$ cause a significantly non-Gaussian character of the distribution of matrix elements [10-12]. Moreover, various statistics of unitary matrices from the different circular ensembles depend strongly on the matrix size, and for small $N$ differ a great deal from the asymptotic large $N$ properties. This is in contrast to the Gaussian ensembles of Hermitian matrices [2] which tend to display lesser sensitivity to the matrix dimension. 
For example, the normalized level spacing distribution $P(S)$ suffers a cut-off at $S=N$, and is close to the Wigner-like distributions only for $N$ upwards of roughly 10 . Moreover, Baranger and Mello [13] have shown that the distribution of transmission intensities $P(T)$ is non-Gaussian for $N=2$ and 4, whereas coming close to Gaussian for larger $N$.

We were led to study the secular polynomials of random unitary matrices in an attempt at constructing semiclassical quasi-energy spectra for the kicked top under conditions of classical chaos. Periodic-orbit theory can be invoked only insofar as periodic orbits are available. In practice, as for any system for which no simple symbolic dynamics is known, one can hope to find all periodic orbits with periods no longer than maybe $n_{\max } \approx 10$, due to the infamous exponential proliferation. These would allow one to semiclassically evaluate traces of powers of the Floquet operator, $\operatorname{tr} F^{n}$, with $n$ up to $n_{\max }$. A Hilbert space of dimension $N=2 n_{\max }$ is then accessible since Newton's formulae [14] (see section 2) allow one to express the first $n_{\max }$ coefficients of the secular polynomial in terms of the first $n_{\max }$ traces; the so-called self-inversiveness of the secular polynomials of unitary matrices [15] then yields the second half of the set of coefficients. The practical applicability of periodic-orbit theory would thus seem severely limited. Inasmuch as the dimension $N$ of the Hilbert space is proportional to the effective size of quantum fluctuations (formally, $N \propto 1 / \hbar)$ one runs out of periodic orbits just when the semiclassical approximation begins to have a raison d'être.

To ease the dilemma just described, one might hope to increase the size of the Hilbert space by throwing dice, according to random-matrix theory, for traces with exponents $n_{\max }<n<N-n_{\max }$. To prepare for such a 'marriage' of semiclassical approximations with random-matrix theory we propose to study here secular polynomials of random unitary matrices from various ensembles. A previous first step in this direction was taken in [16], where the means and the mean squares of the coefficients mentioned were calculated for the CPE and the CUE. A related study was presented by Bogomolny et al $[15,17]$ who gave the distribution of the roots of random self-inverse polynomials.

Even though the bulk of the work to be presented is analytical we performed extensive comparisons with numerical data on ensembles of unitary matrices. To generate the data we constructed COE and CUE matrices with the algorithm given in [18] and CSE matrices as described in [19].

\section{Coefficients of secular polynomials}

\subsection{Theory}

The secular polynomial of a unitary matrix $U$ of size $N$ is defined as

$$
\operatorname{det}(U-\lambda)=\sum_{n=0}^{N}(-\lambda)^{n} a_{N-n}=\prod_{i}\left(\mathrm{e}^{\mathrm{i} \varphi_{i}}-\lambda\right)
$$

where the $\varphi_{i}$ are the eigenphases of $U$ and $a_{0}=1$. We are interested in the statistics of the secular coefficients $a_{n}$ due to various ensembles of random matrices. We shall characterize these ensembles by their joint densities of the $N$ eigenphases $\varphi_{i}$,

$$
d_{N}^{\beta}\left(\varphi_{1}, \varphi_{2}, \ldots, \varphi_{N}\right)=C(\beta, N) \prod_{i<j}\left|\mathrm{e}^{\mathrm{i} \varphi_{i}}-\mathrm{e}^{\mathrm{i} \varphi_{j}}\right|^{\beta}
$$

where $C(\beta, N)$ is a normalization constant while $\beta$, the so-called degree of level repulsion, distinguishes the ensembles: $\beta=0$ for the Poissonian ensemble for which the eigenphases are independently and uniformly distributed over the interval $[0,2 \pi)$; the circular orthogonal, 
unitary, and symplectic ensembles are characterized by, respectively, $\beta=1, \beta=2$, and $\beta=4$. Occasionally we shall allow the parameter $\beta$ to range freely among the real numbers. All of the ensembles in consideration are homogeneous inasmuch they do not distinguish any particular value of any eigenphase. It follows immediately that the ensemble means of all coefficients of the secular polynomial vanish,

$$
\overline{a_{n}}=\int_{0}^{2 \pi} \mathrm{d} \varphi_{1} \ldots \mathrm{d} \varphi_{N} a_{N} d_{N}^{\beta}\left(\varphi_{1}, \varphi_{2}, \ldots, \varphi_{N}\right)=0 .
$$

Next, we are interested in the ensemble averages $\overline{a_{n} a_{m}^{*}}$ which can be obtained from the generating function

$$
\begin{aligned}
P_{N}^{\beta} & =\overline{\operatorname{det}(U-\lambda)(U-\mu)^{\dagger}}=\overline{\prod_{k}\left(\mathrm{e}^{\mathrm{i} \varphi_{k}}-\lambda\right)\left(\mathrm{e}^{\mathrm{i} \varphi_{k}}-\mu\right)^{*}} \\
& =\int_{0}^{2 \pi} \mathrm{d} \varphi_{1} \ldots \mathrm{d} \varphi_{N} d_{N}^{\beta}\left(\varphi_{1}, \varphi_{2}, \ldots, \varphi_{N}\right) \prod_{k}\left(\mathrm{e}^{\mathrm{i} \varphi_{k}}-\lambda\right)\left(\mathrm{e}^{\mathrm{i} \varphi_{k}}-\mu\right)^{*} .
\end{aligned}
$$

For $\lambda=\mathrm{e}^{\mathrm{i} \varphi}, \mu=\mathrm{e}^{\mathrm{i} \chi}$ it follows from the periodicity of the integrand that the integral depends on the phases $\varphi, \chi$ only through the variable $x=\mathrm{e}^{\mathrm{i}(\varphi-\chi)}$. Thus our generating function may be written as

$$
P_{N}^{\beta}(x)=\overline{\prod_{i}\left(\mathrm{e}^{\mathrm{i} \varphi_{i}}-\mathrm{e}^{\mathrm{i} \varphi}\right)\left(\mathrm{e}^{-\mathrm{i} \varphi_{i}}-\mathrm{e}^{-\mathrm{i} \chi}\right)}=\overline{\prod_{i} f\left(\varphi_{i}, x\right)}=\sum_{n=0}^{N} x^{n} \overline{\left|a_{n}\right|^{2}}
$$

where we have introduced the auxiliary function

$$
f(\varphi, x)=\left(\mathrm{e}^{\mathrm{i} \varphi}-x\right)\left(\mathrm{e}^{-\mathrm{i} \varphi}-1\right) .
$$

Hence all correlations $\overline{a_{n} a_{m}^{*}}$ for $m \neq n$ vanish. Moreover, one easily shows

$$
\overline{\left|a_{n}\right|^{2}}=\overline{\left|a_{N-n}\right|^{2}}
$$

which is in accord with the so-called self-inversiveness, $a_{N-n}=a_{n}^{*} a_{N}$ [14]; being a consequence of but slightly weaker than unitarity self-inversiveness entails each root of a polynomial to either lie on the unit circle of the complex plane or to be accompanied by its inverse as another root.

The variances $\overline{\left|a_{n}\right|^{2}}$ are most easily calculated in the Poissonian case $\beta=0$,

$$
P_{N}^{0}(x)=C(0, N)\left[\int_{0}^{2 \pi} \mathrm{d} \varphi f(\varphi, x)\right]^{N}=(1+x)^{N} .
$$

Therefore $\overline{\left|a_{n}\right|^{2}}=\left(\begin{array}{l}N \\ n\end{array}\right)$ for $\beta=0[16]$.

Next, we turn to the case $\beta=2$ which was already treated in [16]. Observing that the function $\prod_{i} f\left(\varphi_{i}, x\right)$ which we want to average is symmetric in the $N$ phases $\varphi_{i}$ and that the density $d_{N}^{2}$ may be written as a product of two Vandermonde determinants we have $[2,3]$

$$
P_{N}^{2}(x)=\int_{0}^{2 \pi} \frac{\mathrm{d}^{N} \varphi}{(2 \pi)^{N}} \operatorname{det}\left(\mathrm{e}^{\mathrm{i}(m-1) \varphi_{m}-\mathrm{i}(n-1) \varphi_{m}}\right) \prod_{k} f\left(\varphi_{k}, x\right)
$$

with $m, n=1,2, \ldots, N$ labelling the rows and columns of the determinant. The integral over $\varphi_{m}$ can now be pulled into the $m$ th row of that determinant whereupon we immediately get

$P_{N}^{2}(x)=\operatorname{det}((1+x) \delta(m-n)-\delta(m-n+1)-x \delta(m-n-1))=\sum_{n=0}^{N} x^{N}$ 
where $\delta(m-n)$ denotes the Kronecker delta. The foregoing generating function entails the variance $\overline{\left|a_{n}\right|^{2}}=1$ for $\beta=2$.

A little more effort is required for the orthogonal and symplectic cases $(\beta=1,4)$. In the orthogonal case we again employ the symmetry of the integrand of the $N$-fold integral in (5) to rewrite that integral, which goes over the hypercube $0 \leqslant \varphi_{1}, \varphi_{2}, \ldots, \varphi_{N} \leqslant 2 \pi$, as $N$ ! times one over the hypertriangle $2 \pi>\varphi_{1}>\varphi_{2}>\cdots>\varphi_{N}>0$. Within the hypertriangle the product of differences takes the form

$\prod_{k<\ell}\left|\mathrm{e}^{\mathrm{i} \varphi_{k}}-\mathrm{e}^{\mathrm{i} \varphi_{\ell}}\right|=\prod_{k<\ell} 2 \sin \left(\frac{\varphi_{k}-\varphi_{\ell}}{2}\right)=\mathrm{i}^{N(N-1) / 2} \operatorname{det}\left(\mathrm{e}^{\mathrm{i} m \varphi_{1}}, \ldots, \mathrm{e}^{\mathrm{i} m \varphi_{N}}\right)$.

The second member in this chain of equated expressions is manifestly positive whereupon the modulus operation can be dropped; the determinant in the last member of (11) has its rows labelled by the parameter $m$ which runs in integer steps between $(N-1) / 2$ and $(N+1) / 2$; the label for the columns is the one on the $N$ integration variables. We may thus write the generating function as

$$
\begin{aligned}
P_{N}^{1}(x)=N ! C & (1, N) \\
& \times \int_{\varphi_{1}>\varphi_{2} \ldots>\varphi_{N}} \mathrm{~d} \varphi_{1} \ldots \mathrm{d} \varphi_{N} \mathrm{i}^{N(N-1) / 2} \operatorname{det}\left(\mathrm{e}^{\mathrm{i} m \varphi_{1}}, \ldots, \mathrm{e}^{\mathrm{i} m \varphi_{N}}\right) \prod_{k} f\left(\varphi_{k}, x\right) .
\end{aligned}
$$

For the symplectic case we find it expedient to extend the $N$-fold integral in (5) to a $(2 N)$-fold one,

$$
\begin{aligned}
P_{N}^{4}(x)=C(4, & N) N ! \int_{\varphi_{1}>\varphi_{2}>\ldots>\varphi_{2 N}} \mathrm{~d} \varphi_{1} \ldots \mathrm{d} \varphi_{2 N} \prod_{k<\ell}^{1 \ldots 2 N}\left(2 \sin \left(\frac{\varphi_{k}-\varphi_{\ell}}{2}\right)\right) \\
\times & \prod_{k=1}^{N}\left[f\left(\varphi_{2 k}, x\right)\left(\frac{-\partial}{\partial \varphi_{2 k-1}}\right) \delta\left(\varphi_{2 k-1}-\varphi_{2 k}-\epsilon\right)\right]
\end{aligned}
$$

where $\epsilon$ is to be sent towards zero from above. To see the equivalence of the foregoing expression to $P_{N}^{4}$ as given by (5) with $\beta=4$ we simply integrate by parts with respect to the phases with odd labels. Only those terms survive for which the $N$ differentiations have turned precisely those $N$ sine functions into cosines which are assigned vanishing arguments by the delta functions; the remaining sine functions then come in quadruples as, symbolically, $s_{13} s_{14} s_{23} s_{24} \rightarrow s_{24}^{4}$. We should note in passing that the delta functions in the foregoing representation of $P_{N}^{4}$ reflect Kramer's degeneracy and that in our definition of the secular polynomial for the symplectic ensemble each of the two-fold degenerate eigenphases appears only once.

The integral representations (12) and (13) are convenient starting points for an explicit evaluation of the generating functions $P_{N}^{1}$ and $P_{N}^{4}$. We propose to start with the slightly easier symplectic case.

In order to actually evaluate the $2 n$-fold integral in (13) we once more employ the identity (11) as extended to $2 N$ ordered phases; we then integrate over every second phase to get rid of the delta functions. Upon renaming the remaining integration variables as $\varphi_{2 k} \rightarrow \varphi_{k}$ and letting the positive infinitesimal $\epsilon$ go to zero we arrive at

$P_{N}^{4}(x)=(-1)^{N} C(4, N) \int_{0}^{2 \pi} \mathrm{d}^{N} \varphi \operatorname{det}\left(m \mathrm{e}^{\mathrm{i} m \varphi_{1}}, \mathrm{e}^{\mathrm{i} m \varphi_{1}}, m \mathrm{e}^{\mathrm{i} m \varphi_{2}}, \mathrm{e}^{\mathrm{i} m \varphi_{2}}, \ldots\right) \prod_{k=1}^{N} f\left(\varphi_{k}, x\right)$

with $|m| \leqslant(2 N-1) / 2$. Note that we have returned to an integral over an $N$-dimensional hypercube, exploiting the symmetry of the integrand. The difficulty to be coped with now lies in the fact that each of the $N$ integration variables appears in a pair of rows of the 
$2 N \times 2 N$ determinant. At this point it is helpful to express that determinant by a Gaussian integral over complex Grassmann variables as

$$
\operatorname{det} M=\int\left(\prod_{k} \mathrm{~d} \eta_{k}^{*} \mathrm{~d} \eta_{k}\right) \exp \left(-\sum_{i, j} \eta_{k}^{*} M_{k l} \eta_{l}\right) .
$$

Accounting for the matrix $M$ from (14) and performing the $2 N$ integrals over the $\eta^{*}$ we arrive at

$$
\begin{aligned}
\int_{0}^{2 \pi} \mathrm{d}^{N} \varphi \operatorname{det} M & =\int\left(\prod_{k} \mathrm{~d} \eta_{k}\right)\left[-\frac{1}{2} \sum_{m, m^{\prime}} A_{m, m^{\prime}} \eta_{m} \eta_{m^{\prime}}\right]^{N} \\
& =N ! \int\left(\prod_{k} \mathrm{~d} \eta_{k}\right) \exp \left[-\frac{1}{2} \sum_{m, m^{\prime}} A_{m, m^{\prime}} \eta_{m} \eta_{m^{\prime}}\right]
\end{aligned}
$$

with the antisymmetric $2 N \times 2 N$ matrix

$$
\begin{aligned}
A_{m m^{\prime}}=\left(m^{\prime}-m\right) \int_{0}^{2 \pi} \mathrm{d} \varphi \mathrm{e}^{\mathrm{i}\left(m+m^{\prime}\right) \varphi} f(\varphi, x) \\
\quad=2 \pi\left(m^{\prime}-m\right)\left((1+x) \delta\left(m+m^{\prime}\right)-\delta\left(m-m^{\prime}+1\right)-x \delta\left(m+m^{\prime}-1\right)\right) .
\end{aligned}
$$

The labelling of the rows and columns of $A$ is inherited from the ordered-phases form of the Vandermonde determinant (11): both $m$ and $m^{\prime}$ run in integer steps from $-(2 N-1) / 2$ to $(2 N-1) / 2$. The remaining Gaussian integral in (16) is easily recognized as the Pfaffian $\sqrt{\operatorname{det} A}$ of the antisymmetric matrix $A$, such that the generating function in search takes the form

$$
P_{N}^{4}(x)=C(4, N) N ! \sqrt{\operatorname{det} A} .
$$

The sign of the Pfaffian $\sqrt{\operatorname{det} A}$ must be chosen such that $P_{N}^{4}(x)$ is positive for positive values of $x$, according to the definition of the generating function.

In a first attempt at evaluating the Pfaffian one may rejoice in the ease in finding it for small values of $N$ which latter suggest a surmise for the general variance,

$$
\overline{\left|a_{n}\right|^{2}}=\left(\begin{array}{l}
N \\
n
\end{array}\right) \frac{1 \times 3 \ldots(2 n-1)}{(2 N-1)(2 N-3) \ldots(2 N-2 n+1)} \quad \text { for } \beta=4 .
$$

This conjecture will be proven in the appendix.

We finally turn to the orthogonal case, taking up the integral representation (12) for $P_{N}^{1}(x)$. For the sake of simplicity let us assume an even dimension $N$. We start with integrating over the angles $\varphi_{i}$ with even indices $i$, pulling the $(2 k)$ th such integral into the $(2 k)$ th row of the determinant; while that integral at first appears as going over the interval $\varphi_{2 k+1}<\varphi_{2 k}<\varphi_{2 k-1}$ we can hurry to replace the lower limit with zero, simply by adding the $N$ th column to the $(N-2)$ th, the resulting $(N-2)$ th to the $(N-4)$ th and so forth, and thus obtain

$$
\begin{array}{r}
P_{N}^{1}(x)=C(1, N) N ! \int_{\varphi_{1}>\varphi_{3}>\ldots>\varphi_{N-1}} \mathrm{~d} \varphi_{1} \mathrm{~d} \varphi_{3} \ldots \mathrm{d} \varphi_{N-1} \mathrm{i}^{N(N-1) / 2} \operatorname{det}\left(\mathrm{e}^{\mathrm{i} m \varphi_{1}} f\left(\varphi_{1}, x\right),\right. \\
\left.\times \int_{0}^{\varphi_{1}} \mathrm{~d} \varphi \mathrm{e}^{\mathrm{i} m \varphi} f(\varphi, x), \mathrm{e}^{\mathrm{i} m \varphi_{3}} f\left(\varphi_{3}, x\right), \int_{0}^{\varphi_{3}} \mathrm{~d} \varphi \mathrm{e}^{\mathrm{i} m \varphi} f(\varphi, x), \ldots\right) .
\end{array}
$$

Now the integrand is symmetric in the remaining $N$ integration variables whereupon we may extend the integration range to the $N$-dimensional hypercube of edge length $2 \pi$ and make up by dropping the factor $N$ !, as we have previously done in (14). The analogy with (14) in fact goes much further: once more, every integration variable appears in two rows 
of the determinant. Going through precisely the same reasoning as before we again incur a Pfaffian form,

$$
P_{N}^{1}(x)=C(1, N) N ! \sqrt{\operatorname{det} B}
$$

with the slightly more unpleasant antisymmetric matrix

$$
B_{m m^{\prime}}=-\mathrm{i} \int_{0}^{2 \pi} \mathrm{d} \varphi \mathrm{d} \varphi^{\prime} f(\varphi, x) f\left(\varphi^{\prime}, x\right) \mathrm{e}^{\mathrm{i}\left(m \varphi+m^{\prime} \varphi^{\prime}\right)} \operatorname{sign}\left(\varphi-\varphi^{\prime}\right) .
$$

Here, the sign function ensures the antisymmetry of the matrix $B$ with $|m| \leqslant(N-1) / 2$. Again, the Pfaffian suggests, by its easily evaluated form for small dimensions $N$, a guess for the variances,

$$
\overline{\left|a_{n}\right|^{2}}=1+\frac{n(N-n)}{N+1} \quad \text { for } \beta=1
$$

We refer to the appendix for the proof of that surmise for $N$ even or odd.

Upon inspecting the variances found above for $\beta=0,2$ and conjectured for $\beta=1,4$ we were led to extrapolate to arbitrary non-negative $\beta$ as

$$
\begin{aligned}
\overline{\left|a_{n}\right|^{2}} & =\left(\begin{array}{l}
N \\
n
\end{array}\right) \frac{1(1+\beta / 2)(1+\beta) \ldots(1+(n-1) \beta / 2)}{(1+(N-1) \beta / 2)(1+(N-2) \beta / 2) \ldots(1+(N-n) \beta / 2)} \\
& =\left(\begin{array}{l}
N \\
n
\end{array}\right) \frac{\Gamma(n+2 / \beta) \Gamma(N-n+2 / \beta)}{\Gamma(2 / \beta) \Gamma(N+2 / \beta)} .
\end{aligned}
$$

This expression has poles at $\beta=-2 /(N-1),-2 /(N-2), \ldots,-2 /(N-n)$ and zeros at $\beta=-2 / 1,-2 / 2,-2 / 3, \ldots,-2 /(n-1)$ for $n \leqslant N / 2$. It is thus analytic and positive for all positive $\beta$, and it goes to zero for $\beta \rightarrow \infty$. This looks like strong evidence for the general validity claimed before. We shall actually turn the conjecture into a theorem in the appendix. The proof will be based on the fact that (24) is equivalent to the differential equation

$\frac{\partial}{\partial x}\left(1+\frac{\beta}{2}\left(N-x \frac{\partial}{\partial x}\right)\right) P_{N}^{\beta}(x)=\left(N-x \frac{\partial}{\partial x}\right)\left(1+\frac{\beta}{2} x \frac{\partial}{\partial x}\right) P_{N}^{\beta}(x)$

for the generating function $P_{N}^{\beta}(x)$.

\subsection{Numerical results}

We constructed random unitary matrices $U$ of different sizes according to the algorithm developed in [18] for the CUE and the COE and later generalized for the CSE [19]. For each such matrix we calculated a complete set of $N$ secular coefficients $a_{n}$ by first computing the traces of arbitrary powers, $t_{n}=\operatorname{tr}\left(U^{n}\right)$, via either matrix multiplication or diagonalization; Newton's formulae [14] (see next section) then led to the $a_{n}$.

Precise estimates of the variance of any random variable require a much larger sample than estimates of the mean. We therefore present numerical results obtained for large samples of relatively small random matrices $(N \sim 20)$, although some computations performed for $N \sim 200$ provide similar results.

Our above formulae for the variance $\overline{\left|a_{n}\right|^{2}}$ of the secular coefficients involve the index $n$ of the coefficient and the matrix size $N$. The dependence on $N$ saturates for large $N$. Figure 1 shows the dependence on $n$ for a fixed matrix size. Due to the property (7) of self-inversiveness this curve is symmetric about $n=N / 2$.

The data of all three ensembles coincide (up to a statistical error) with the theoretical predictions. Note that the width of the distribution of coefficients decreases with increasing 


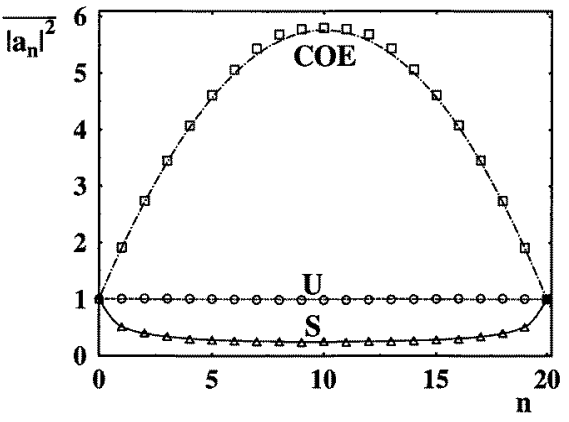

Figure 1. Variance of the distribution of the $n$th coefficient $\overline{\left|a_{n}\right|^{2}}$ obtained from 40000 matrices of size $N=20$ typical of COE $(\square)$, CUE $(O)$, and CSE $(\triangle)$. The lines represent our analytical results.

degrees of repulsion and is smallest for the symplectic ensemble. Additional numerical investigations confirmed the expectation that the phases of the $a_{n}$ are distributed uniformly in the range $[0,2 \pi)$ for any canonical ensemble.

Even though the formula (24) for the variance is primarily meant to cover the four canonical ensembles $\beta=0$ (CPE), 1 (COE), 2 (CUE), and 4 (CSE), we could not resist the temptation to test its usefulness for intermediate cases. To this end we constructed an ensemble of unitary matrices interpolating between the Poisson and unitary ensembles according to the method presented in [20]. This intermediate ensemble depends on one control parameter $\delta$, varying from 0 (CPE) to 1 (CUE). Figure 2 shows the dependence of the variance of the first two coefficients on the matrix size $N$ for $\delta$ equal to $0.0,0.2,0.4$, 0.7 , and 1.0. For each case the value of the parameter $\beta$ chosen to fit the $N$ dependence of $\overline{\left|a_{1}\right|^{2}}$, inserted into (24), provides a fair approximation for $\overline{\left|a_{2}\right|^{2}}$, and similarly for subsequent coefficients. This astonishing fact reveals a certain validity of the general formula (24) with non-integer values of $\beta$ for ensembles in between the usual universality classes.

\section{Traces of powers of matrices from circular ensembles}

The characteristic polynomial of a matrix $U$ is related to the traces of its powers

$$
t_{n}=\operatorname{tr}\left(U^{n}\right)=\sum_{i=1}^{N} \mathrm{e}^{\mathrm{i} n \varphi_{i}}
$$

by

$$
\operatorname{det}(U-\lambda)=(-\lambda)^{N} \exp \left(\operatorname{tr} \ln \left(1-\frac{1}{\lambda} U\right)\right) .
$$

Expanding both sides in powers of $\lambda$ one finds the explicit relations between the $a_{n}$ and the $t_{n}$ which were already established by Newton [14]. A compact representation is

$$
a_{n}=\frac{1}{n !}\left|\begin{array}{cccccc}
t_{1} & 1 & 0 & 0 & \cdots & 0 \\
t_{2} & t_{1} & 2 & 0 & \cdots & 0 \\
t_{3} & t_{2} & t_{1} & 3 & \cdots & 0 \\
\cdots & \cdots & \cdots & \cdots & \cdots & \cdots \\
t_{n} & t_{n-1} & t_{n-2} & t_{n-3} & \cdots & t_{1}
\end{array}\right| .
$$

We infer from (27) that there are only $N$ independent traces $t_{1}, \ldots, t_{N}$. Moreover, for unitary matrices for which the eigenphases $\varphi_{i}$ are real, the number of independent complex parameters is again reduced by a factor of a half. The first $N / 2$ traces suffice to determine all $N$ coefficients $a_{n}$ (cf (7)). 

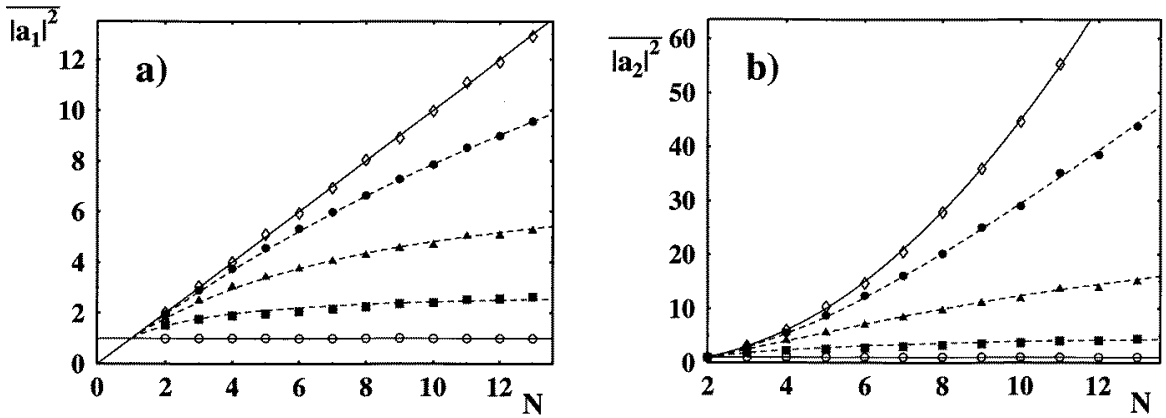

Figure 2. Variance of coefficients for crossover CPE-CUE: (a) $\overline{\left|a_{1}\right|^{2}}$ and (b) $\overline{\left|a_{2}\right|^{2}}$ as functions of the matrix size $N$. Control parameter $\delta$ is equal to 0.0 (Poisson, $\diamond), 0.2(\bullet), 0.4,0.7(\mathbf{\square})$, and 1.0 (CUE, $\bigcirc$ ), while the level-repulsion parameter $\beta$ fitted simultaneously for both coefficients equals $0.0,0.06,0.24,0.69$, and 2.0 , respectively.

Clearly, the $t_{n}$ all vanish in the mean for all of the circular ensembles considered here, due to the uniformity of the distribution of the $\varphi_{i}$. For a more complete characterization of the statistics of the traces we propose to calculate their marginal probability densities

$$
P_{N, n}^{\beta}(t)=\overline{\delta^{2}\left(t-\sum_{i=1}^{N} \mathrm{e}^{\mathrm{i} n \varphi_{i}}\right)}
$$

where $\delta^{2}(t)$ is a two-dimensional delta function in the complex $t$ plane; the ensemble average is to be performed with the weight (2). It turns out to be convenient to first calculate the Fourier transform

$$
\hat{P}_{N, n}^{\beta}(k)=\overline{\exp \left(-\frac{1}{2} \mathrm{i} \sum_{i}\left(k \mathrm{e}^{-\mathrm{i} n \varphi_{i}}+k^{*} \mathrm{e}^{+\mathrm{i} n \varphi_{i}}\right)\right)} .
$$

Due to the periodicity of all functions of the phases $\varphi_{i}$ involved, the characteristic function $\hat{P}_{N, n}^{\beta}(k)$ depends on $k$ only through the modulus $|k|$ while the density $P_{N, n}^{\beta}(t)$ is only a function of $|t|$. Henceforth we assume $k=k^{*}=|k|$ and write

$$
\hat{P}_{N, n}^{\beta}(k)=\overline{\exp \left(-\mathrm{i} k \sum_{i} \cos \left(n \varphi_{i}\right)\right)} .
$$

We immediately obtain for the Poissonian ensemble

$$
\hat{P}_{N, n}^{0}(k)=J_{0}(k)^{N}
$$

$J_{0}(k)$ being a Bessel function. It follows that the densities $P_{N, n}^{0}$ are the same for all values of the exponent $n$, a rather intuitive result given the statistical independence of the phases $\varphi_{i}$ in the Poissonian case. Equally expected for such a Poissonian random walk is the independence of the mean squared 'displacement' of $n, \overline{\left|t_{n}\right|^{2}}=N$.

For the unitary ensemble, $\beta=2$, we exploit the symmetry of the exponential to be averaged in the phases $\varphi_{i}$ and employ the analogue of (9) and find the characteristic function to take the form of a Toeplitz determinant,

$$
\hat{P}_{N, n}^{2}(k)=\operatorname{det}\left(\int_{0}^{2 \pi} \frac{\mathrm{d} \varphi}{2 \pi} \mathrm{e}^{\mathrm{i} \varphi(\ell-m)} \mathrm{e}^{-\mathrm{i} k \cos (n \varphi)}\right) \quad \ell, m=1, \ldots, N .
$$


The remaining $\varphi$ integral again yields Bessel functions and can be written as

$$
\hat{P}_{N, n}^{2}(k)=\operatorname{det}\left(\sum_{s=-\infty}^{+\infty} J_{|s|}(k)(-\mathrm{i})^{|s|} \delta(\ell-m+n s)\right) .
$$

Clearly, the number of non-zero elements of the $N \times N$ determinant here incurred decreases as the order $n$ of the trace $t_{n}$ in consideration grows. In particular, for $n \geqslant N$ only the diagonal elements are non-zero such that

$$
\hat{P}_{N, n}^{2}(k)=J_{0}(k)^{N} \quad \text { for } n \geqslant N .
$$

At the other extreme, $n=1$, we meet with the full Toeplitz determinant

$$
\hat{P}_{N, 1}^{2}(k)=T_{N}=\left|\begin{array}{cccc}
J_{0} & -\mathrm{i} J_{1} & -J_{2} & \cdots \\
-\mathrm{i} J_{1} & J_{0} & -\mathrm{i} J_{1} & \cdots \\
-J_{2} & -\mathrm{i} J_{1} & J_{0} & \cdots \\
\vdots & & &
\end{array}\right| .
$$

Intermediate values of $n$ lead to the subdeterminants $T_{m}$ obtained by cancelling the last $N-m$ rows and columns of $T_{N}$,

$$
\begin{array}{ll}
\hat{P}_{N, n}^{2}(k)=T_{2}^{N-n} T_{1}^{2 n-N} & \text { for } N \geqslant n \geqslant \frac{1}{2} N \\
\hat{P}_{N, n}^{2}(k)=T_{3}^{N-2 n} T_{2}^{3 n-N} & \text { for } \frac{1}{2} N \geqslant n \geqslant \frac{1}{3} N
\end{array}
$$

and so forth. This can be seen as follows. Starting with $N \geqslant n \geqslant \frac{1}{2} N$ one checks that the determinant in (34) has non-vanishing elements residing only in the diagonal, $J_{0}(k) \delta(\ell-m)$, and in two subdiagonals, $-\mathrm{i} J_{1}(k) \delta(\ell-m+n)$. One moves the $(1+n)$ th row to become the second, then the $(1+n)$ th column to become the second and thus isolates a $2 \times 2$ block $T_{2}=J_{0}(k)^{2}+J_{1}(k)$ in the upper left corner. One repeats this process by moving the $(2+n)$ th rows and columns to become the fourth and so forth until one arrives at a block diagonal determinant in which the $2 \times 2$ block $T_{2}$ and the $1 \times 1$ block $T_{1}=J_{0}(k)$ appear $N-n$ times and $2 n-N$ times, respectively. The procedure for $\frac{1}{2} N \geqslant n \geqslant \frac{1}{3} N$ is analogous: one moves the $(1+n)$ th and the $(2+n)$ th row to become the second and third, respectively, then does likewise to the $(1+n)$ th and $(2+n)$ th column and thus generates the $3 \times 3$ block $T_{3}$ and so forth. As $n$ decreases towards unity we meet all the

$$
\hat{P}_{N, n}^{2}(k)=T_{m+1}^{N-m n} T_{m}^{(m+1) n-N} \quad \text { for } N / m \geqslant n \geqslant N /(m+1)
$$

with $m=1, \ldots, N$.

Simplest to deal with is, of course, the case of the smallest non-trivial dimension, $N=2$, and there we obtain

$$
\hat{P}_{2,1}^{2}(k)=J_{0}^{2}(k)+J_{1}^{2}(k) \quad \hat{P}_{2,2}^{2}(k)=J_{0}^{2}(k) .
$$

By Fourier transforming we produce the densities of the first and second trace,

$$
P_{2,1}^{2}(t)=\frac{\sqrt{4-|t|^{2}}}{2 \pi^{2}|t|} \quad P_{2,2}^{2}(t)=\frac{1}{\pi^{2}|t| \sqrt{4-|t|^{2}}} .
$$

Now we propose to show that the distribution $P_{N, n}^{2}$ of the $n$th trace behaves like a Gaussian with respect to its moments $\overline{\left|t_{n}\right|^{2 m}}$ for sufficiently low orders. To this end we define the functions

$$
\tilde{J}_{m}(k)=(-\mathrm{i} k)^{m} J_{m}(k)
$$

which have the property (Abramowitz and Stegun 9.1.30 [21])

$$
\frac{\mathrm{i}}{k} \frac{\partial}{\partial k} \tilde{J}_{m}(k)=\tilde{J}_{m-1}(k) \text {. }
$$


The Toeplitz determinant $T_{N}$ can then be rewritten as

$$
T_{N}=\operatorname{det}\left(\tilde{J}_{m}, \tilde{J}_{m-1}, \ldots\right) \quad \text { with } m=0, \ldots, N-1 .
$$

From this we find with (41) and (42) for $l \leqslant N$

$$
\left.\left(\frac{1}{k} \frac{\partial}{\partial k}\right)^{l} T_{N}\right|_{k=0}=\left(\frac{-1}{2}\right)^{l} \text {. }
$$

To prove the foregoing identity we proceed as follows. Applying $(1 / k)(\partial / \partial k)$ once to $T_{N}$ we get a determinant differing from $T_{N}$ only in the last column where, according to (42), $\tilde{J}_{m-N+1} \rightarrow \tilde{J}_{m-N}$. Now setting $k=0$ and invoking $\tilde{J}_{m}(0)=0$ for positive integer $n$ while $\tilde{J}_{m}(0)=\left(-\frac{1}{2} \mathrm{i}\right)^{m} /(-m)$ ! for $m=0,-1,-2, \ldots$ we face a determinant with vanishing elements below the diagonal and thus equalling the product of its diagonal elements; among these only the last, $\tilde{J}_{1}(0)=-\frac{1}{2} \mathrm{i}$, differs from unity whereupon (44) is proven for $\ell=1$. In the next step, $\ell=2, T_{N}$ is changed such that only the elements in the last two columns may get their indices shifted; again setting $k=0$ we are left with a $2 \times 2$ determinant which yields (44) for $\ell=2$. In the $\ell$ th step we get a sum of $\ell \times \ell$ determinants which is evidently independent of the dimension $N$ for $\ell \leqslant N$. Due to that independence we need not pursue the non-trivial task of proving (44) for arbitrary $\ell \leqslant N<\infty$ but rather invoke the much more easily proven result, to be established in the next section, that for $N \rightarrow \infty$ the determinant $T_{N}$ is a Gaussian in $k$. It follows that for finite $N$ the expansion of $T_{N}$ in powers of $k^{2}$ coincides with that of $\exp \left(-k^{2} / 4\right)$ up to the $N$ th power. Equations (35)-(37) show that $P_{N, n}^{2}(t)$ behaves like a Gaussian distribution with respect to all moments $\overline{\left|t_{n}\right|^{2 m}}$ of orders $m \leqslant N / n$. In particular, we find for the variances of the traces

$$
\overline{\left|t_{n}\right|^{2}}= \begin{cases}n & \text { for } 0<n \leqslant N \\ N & \text { for } n \geqslant N .\end{cases}
$$

Interestingly, these variances grow towards the Poissonian value $N$ as $n \rightarrow N$ from below and then remain stuck as $n$ grows further.

We now proceed to the orthogonal and symplectic cases. Starting as in the previous section with integrating over alternating variables we find for the orthogonal case with even $N$ and the symplectic case

$$
\begin{array}{ll}
\hat{P}_{N, n}^{1}(k) \propto(\operatorname{det} A)^{1 / 2} & \text { for } \beta=1 \\
\hat{P}_{N, n}^{4}(k) \propto(\operatorname{det} B)^{1 / 2} & \text { for } \beta=4
\end{array}
$$

with the antisymmetric matrices

$$
\begin{aligned}
A_{m m^{\prime}} & =\sum_{s s^{\prime}} J_{|s|}(k) J_{\left|s^{\prime}\right|}(k) \frac{(-\mathrm{i})^{|s|}(-\mathrm{i})^{\left|s^{\prime}\right|}}{m+n s} \\
& \times \delta\left(m+m^{\prime}+n\left(s+s^{\prime}\right)\right)
\end{aligned}
$$

We have not proven the Gaussian property but have calculated the variances. In the orthogonal case $(\beta=1)$ we find

$$
\overline{\left|t_{n}\right|^{2}}= \begin{cases}2 n-n \sum_{m=1}^{n} \frac{1}{m+(N-1) / 2} & \text { for } 0<n \leqslant N \\ 2 N-n \sum_{m=1}^{N} \frac{1}{m+n-(N+1) / 2} & \text { for } n \geqslant N\end{cases}
$$




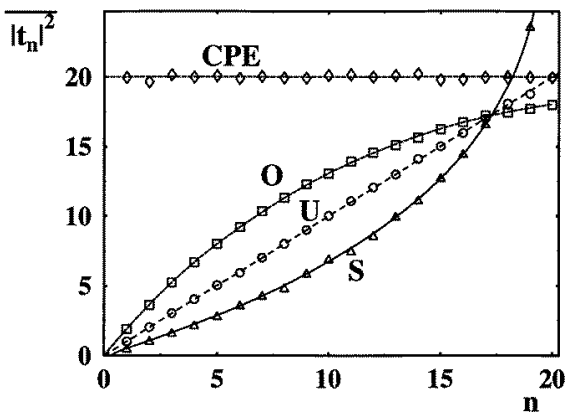

Figure 3. Mean squared traces $\overline{\left|t_{n}\right|^{2}}$ for $10^{4}$ matrices of size $N=20$ typical of $\operatorname{CPE}(\diamond), \operatorname{COE}(\square)$, CUE $(O)$, and $\operatorname{CSE}(\triangle)$ compared with analytical results (curves).

while the symplectic case $(\beta=4)$ yields

$$
\overline{\left|t_{n}\right|^{2}}= \begin{cases}\frac{1}{2} n+\frac{1}{4} n \sum_{m=1}^{n} \frac{1}{N+\frac{1}{2}-m} & \text { for } 0<n \leqslant 2 N \\ N & \text { for } n \geqslant 2 N .\end{cases}
$$

Needless to say, these mean squared traces could have been read off the well known two-level correlation functions of the circular ensembles the Fourier transforms of which our variances in essence are [2]. Indeed, by introducing a non-normalized density of eigenphases as

$$
\rho(\varphi)=2 \pi \sum_{i=1}^{N} \delta\left(\varphi-\varphi_{i}\right)=\sum_{i=1}^{N} \sum_{n=-\infty}^{\infty} \mathrm{e}^{-\mathrm{i} n\left(\varphi-\varphi_{i}\right)}
$$

one immediately sees that the two-point correlation function of that density reads

$$
\overline{\rho(\varphi) \rho\left(\varphi^{\prime}\right)}=\sum_{n=-\infty}^{\infty} \overline{\left|t_{n}\right|^{2}} \mathrm{e}^{-\mathrm{i} n\left(\varphi-\varphi^{\prime}\right)} .
$$

Figure 3 reveals excellent agreement of the mean squared traces as computed for samples of $4 \times 10^{4} 20 \times 20$ matrices of the four ensembles considered with the analytical predictions. Note that for the Poisson circular ensemble $\overline{\left|t_{n}\right|^{2}}$ equals the matrix size $N$, without dependence on $n$. For small $n$ the variance of traces decreases with the repulsion parameter $\beta$. The data for the symplectic ensemble are obtained with $2 N$-dimensional matrices, which provide $N$ different eigenvalues each.

Figure 4 displays the similarly fine agreement of our analytical results for the densities $P_{N, n}^{2}$ with numerical data for sets of random matrices drawn from the circular unitary ensemble. This demonstrates that for finite $n$ and large $N$ the distributions are Gaussians. We have performed similar numerical studies for the orthogonal and symplectic ensembles, again finding Gaussian marginal distributions for the traces of sufficiently large matrices. Moreover, in all cases studied, the distribution of the traces was isotropic, i.e. without any phase dependence.

We should add a word of intuitive explanation to the statistics of the traces for large dimensions $N$. The $n$th trace of a unitary matrix may be thought of as a random walk in the complex plane, with each of the $N$ steps of unit length and the $i$ th step in a direction given as $n$ times the $i$ th eigenphase $\varphi_{i}$. These directions are mutually independent and uniformly distributed for the Poissonian ensemble which fact explains the independence of the characteristic function (32) of $n$ and the ensuing first and second moments, $\overline{t_{n}}=0, \overline{\left|t_{n}\right|^{2}}=N$. In accordance with the central limit theorem the rescaled traces $t_{N} / \sqrt{N}$ tend, with $N \rightarrow \infty$, to have a Gaussian distribution of zero mean and unit 


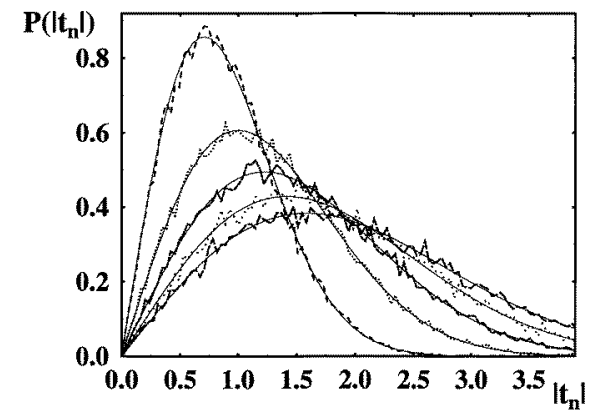

Figure 4. Distribution of the moduli of the first five traces $\left|t_{1}\right|, \ldots,\left|t_{5}\right|$ for $10^{5}$ CUE matrices of size $N=20$. Narrower full curves correspond to Gaussian distributions with appropriate variances $\overline{\left|t_{n}\right|^{2}}=n$.

variance. Without such rescaling, the moments $\overline{\left|t_{n}\right|^{2 m}}$ with $m \ll N$ differ from those of the Gaussian defined by the first two moments only by corrections of relative order $1 / N$.

For the circular ensembles with $\beta>0$ the phases $\varphi_{i}$ display repulsion of degree $\beta$ such that the directions of subsequent steps in the random walk mentioned are not independent. The correlations between the phases cannot prevent near-Gaussian behaviour of the traces $t_{n}$ with $n \ll N$, as is intuitive in view of the local character of the spectral correlations. Moreover, while the phases $\varphi_{i}$ cover the interval $[0,2 \pi)$ uniformly once, their multiples $n \varphi_{i}$ go around that interval $n$ times such that for $n>1$ the phases $n \varphi_{i}[\bmod (2 \pi)]$ may exhibit accidental close neighbourhoods of originally distant $\varphi_{i}$.

\section{Joint density of traces for large CUE matrices}

We shall employ here a powerful theorem about determinants of Toeplitz matrices, due originally to Szegö and Kac and extended by Hartwig and Fisher [22], to find the marginal and joint distributions of the traces $t_{n}$ of CUE matrices in the limit of large dimension $N$. As our starting point we recall the identity (9) for the CUE average of a symmetric function of all $N$ phases. Assuming, moreover, that symmetric function to have the form of a product we can pull the integral over the $m$ th phase $\varphi_{m}$ into the $m$ th row of the determinant in (9) and thus express the average as a Toeplitz determinant,

$\overline{\prod_{m=1}^{N} f\left(\varphi_{m}\right)}=\operatorname{det}\left(f_{m}, f_{m-1}, \ldots, f_{-N+1}\right)=T(\{f\}) \quad m=0,1, \ldots, N-1$

the elements of which are given by the Fourier transform

$$
f_{m}=\int_{0}^{2 \pi} \frac{\mathrm{d} \varphi}{2 \pi} \mathrm{e}^{-\mathrm{i} m \varphi} f(\varphi)
$$

of the function $f(\varphi)$. We had incurred two examples in (9), (10) and (33), (34). The theorem in question says that for large $N$ the above determinant is given by

$$
\ln T(\{f\})=N l_{0}+\sum_{n=0}^{\infty} n l_{n} l_{-n}
$$

where the $l_{n}$ are the Fourier coefficients of $\ln f(\varphi)$, i.e. $\ln f(\varphi)=\sum_{n=-\infty}^{\infty} l_{n} \mathrm{e}^{\mathrm{i} n \varphi}$. The conditions the function $f(\varphi)$ must meet for the above limiting form to hold are (i) $f(\varphi) \neq 0$ for $0 \leqslant \varphi<2 \pi$, (ii) $\arg f(2 \pi)=\arg f(0)$, (iii) $\sum_{n=-\infty}^{\infty}\left|f_{n}\right|<\infty$, and (iv) $\sum_{n=-\infty}^{\infty}|n|\left|f_{n}\right|^{2}<\infty$; they are fulfilled in all examples of interest here. 
In a first application we return to the Toeplitz determinant (34) which gives the density $P_{N, n}^{2}$ of the $n$th trace. The only non-vanishing Fourier coefficients of $\ln f(\varphi)=-\mathrm{i} k \cos n \varphi$ are $l_{ \pm n}=-\mathrm{i} k / 2$ whereupon we get the Gaussian anticipated in the previous section,

$$
\hat{P}_{N, n}^{2}(k)=\mathrm{e}^{-n k^{2} / 4} \Longleftrightarrow P_{N, n}^{2}(t)=\frac{1}{n \pi} \mathrm{e}^{-|t|^{2} / n} \quad \text { for } N \gg 1 .
$$

No more difficult to obtain is the joint density of the first $n$ traces

$$
P_{N}^{\beta}\left(t_{1}, \ldots, t_{n}\right)=\overline{\prod_{m=1}^{n} \delta^{2}\left(t_{m}-\sum_{i=1}^{N} \mathrm{e}^{\mathrm{i} m \varphi_{i}}\right)}
$$

since its Fourier transform $\hat{P}_{N}^{2}\left(k_{1}, \ldots, k_{n}\right)$ is once more of the form (52) with

$$
f(\varphi)=\exp \left(-\frac{1}{2} \mathrm{i} \sum_{m=1}^{n}\left(k_{m} \mathrm{e}^{-\mathrm{i} m \varphi}+k_{m}^{*} \mathrm{e}^{\mathrm{i} m \varphi}\right)\right) .
$$

The non-vanishing Fourier coefficients of the logarithm of that latter function are $l_{m}=$ $-\mathrm{i} k_{m} / 2, l_{-m}=-\mathrm{i} k_{m}^{*} / 2$ with $m=1, \ldots, n$. The theorem (54) thus yields, for $N \gg n$,

$$
\begin{gathered}
\hat{P}_{N}^{2}\left(k_{1}, \ldots, k_{n}\right)=\exp \left(-\sum_{m=1}^{n} m\left|k_{m}\right|^{2} / 4\right) \Longleftrightarrow P_{N}^{2}\left(t_{1}, \ldots, t_{n}\right) \\
=\frac{1}{n ! \pi^{n}} \exp \left(-\sum_{m=1}^{n}\left|t_{m}\right|^{2} / m\right)
\end{gathered}
$$

i.e. the product of the marginal distributions of the first $n$ traces. The result generalizes in an obvious way to the joint density of an arbitrary set of finite-order traces. We thus conclude that in the limit $N \rightarrow \infty$ the finite-order traces are statistically independent and all have Gaussian distributions.

We can now briefly comment on the conditions of applicability of the Hartwig-Fisher theorem given above. The first two of them are clearly fulfilled here since i $\ln f(\varphi)$ as given by (57) is real, continuous, and periodic. The third and fourth conditions are met since the derivative $f^{\prime}(\varphi)=\sum_{m=-\infty}^{\infty} \mathrm{i} m f_{m} \mathrm{e}^{\mathrm{i} m \varphi}$ is square integrable (trivially indeed since $\mathrm{i} \ln f(\varphi)$ is a finite Fourier series); in particular, $f^{\prime}(\varphi)$ obeys Parseval's identity,

$$
\sum_{m=-\infty}^{\infty}|m|^{2}\left|f_{m}\right|^{2}=\int_{0}^{2 \pi}\left|f^{\prime}(\varphi)\right|^{2} \mathrm{~d} \varphi=\pi \sum_{m=1}^{n} m^{2}\left|k_{m}\right|^{2}<\infty .
$$

Since $|m|\left|f_{m}\right|^{2} \leqslant|m|^{2}\left|f_{m}\right|^{2}$ it follows that $\sum_{m=-\infty}^{\infty}|m|\left|f_{m}\right|^{2}<\infty$, i.e. the validity of condition (iii).

Finally, we invoke Cauchy's inequality in

$$
\sum_{m=-\infty}^{\infty}\left|f_{m}\right|=\left|f_{0}\right|+\sum_{m \neq 0}^{\infty}\left|\frac{1}{m}\right|\left|m f_{m}\right| \leqslant\left|f_{0}\right|+\left(\sum_{m \neq 0}^{\infty}\left|\frac{1}{m}\right|^{2}\right)^{1 / 2}\left(\sum_{m \neq 0}^{\infty}\left|m f_{m}\right|^{2}\right)^{1 / 2} .
$$

Upon using (59) and the convergence of $\sum_{m \neq 0}^{\infty} 1 /|m|^{2}$ we verify condition (iv).

For finite dimension the independence as well as the Gaussian character of the traces are only approximate. For sets of traces both of these properties tend to get lost as the sum of the orders of the traces in a set increases. In particular, since all traces $t_{n}$ are uniquely determined by the $N$ real eigenphases, only $\frac{1}{2} N$ traces can be independent.

Preliminary numerical studies suggest that the finite-order traces might be similarly independent and Gaussian for the COE and the CSE. For the CPE, of course, the independence holds trivially. 


\section{Remarks on the distributions of coefficients of secular polynomials}

As we have just seen the first few traces $t_{1}, t_{2}, \ldots$ of large unitary matrices drawn from any of the circular ensembles display no noticeable correlations. It follows that the coefficients $a_{1}, a_{2}, \ldots$ must bear strong mutual correlations simply since $a_{n}$ can be expressed in terms of the first $n$ traces through Newton's formulae (25). One must therefore expect that the explicit form of the marginal and joint distributions of the $a_{n}$ are hard to come by. An exception is provided by $a_{1}$, the marginal distribution of which is trivially related to that of the first trace since $a_{1}=t_{1}$.

A slightly less trivial result may be obtained for the distribution $P\left(a_{2}\right)$ of the second coefficient. Since $a_{2}=\frac{1}{2}\left(t_{1}^{2}-t_{2}\right)$ we may invoke the CUE joint distribution (58) of the first two traces to get

$$
P\left(a_{2}\right)=\sqrt{\frac{2 e}{\pi^{3}}} \int_{0}^{2} \frac{\mathrm{d} x}{\sqrt{x(2-x)}} \exp \left(-\frac{1}{x}-\left|a_{2}\right|^{2} x\right) .
$$

A saddle-point approximation to the foregoing integral immediately reveals that $P\left(a_{2}\right)$ decays exponentially for large $a_{2}$. Proceeding similarly one may combine Newton's formulae with the joint distribution of the traces to get the marginal and joint distributions of the first few $a_{n}$, with decreasingly compact and enjoyable results.

Figure 5 presents the distributions of some moduli $\left|a_{n}\right|$ as obtained numerically from $10^{5}$ CUE matrices of the size $10 \times 10$. For $n=2$ these numerical data agree well with the distribution $2 \pi\left|a_{2}\right| P\left(a_{2}\right)$ according to (61). All of those curves grow linearly out of the origin with a slope increasing with the index $n$ of the coefficient $a_{n}$. On the other hand, all of these distributions but the first are characterized by a long exponential tail. The latter originates from the convolution type integrals which combine the densities of the traces to those of the coefficients; it contrasts with the Gaussian tail of the distribution of the first coefficient. The qualitative features just outlined for the CUE are shared by the distributions $P\left(a_{n}\right)$ for the other canonical ensembles.

\section{Comparison with a dynamical system}

We propose here to examine how far our results on distributions of the coefficients $a_{n}$ and traces $t_{n}$ of matrices, drawn from the canonical circular ensembles, are applicable to dynamical systems. Choosing the familiar model of the periodically kicked top [3,23] we work with a Floquet operator of the form

$$
\begin{gathered}
F=\exp \left(-\mathrm{i} \frac{k_{x}}{2 j+1} J_{x}^{2}-\mathrm{i} p_{x} J_{x}\right) \exp \left(-\mathrm{i} \frac{k_{y}}{2 j+1} J_{y}^{2}-\mathrm{i} p_{y} J_{y}\right) \\
\times \exp \left(-\mathrm{i} \frac{k_{z}}{2 j+1} J_{z}^{2}-\mathrm{i} p_{z} J_{z}\right) .
\end{gathered}
$$

This involves the components of an angular momentum operator $J_{x}, J_{y}, J_{z}$ which satisfy standard commutation relations, $\left[J_{x}, J_{y}\right]=\mathrm{i} J_{z}$ etc. The quantum number $j$ fixes $\boldsymbol{J}^{2}=$ $j(j+1)$ and the size of the Hilbert space, $N=2 j+1$.

For generic values of the parameters $k_{x}, k_{y}, k_{z}$ and $p_{x}, p_{y}, p_{z}$ the corresponding classical dynamics is chaotic and there is no geometric nor anti-unitary symmetry left [23]. All previously studied statistical properties of the quasi-energy spectrum and the eigenvectors were found to be remarkably faithful to the predictions of the CUE [3]. On the other hand, when the parameters $k_{y}$ and $p_{y}$ (or, instead, $k_{x}$ and $p_{x}$ ) are set to zero, an anti-unitary symmetry under time reversal appears, and in this case the spectral and eigenvector statistics 


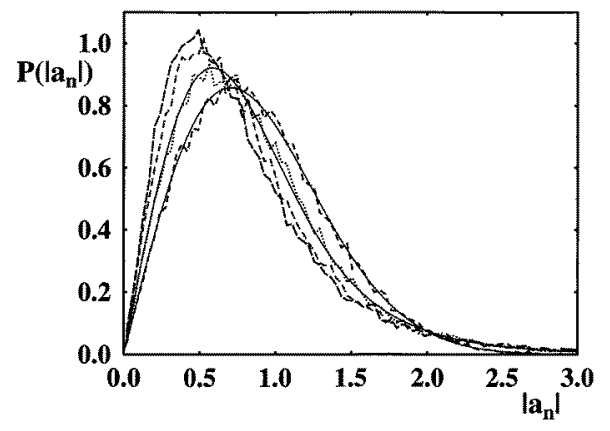

Figure 5. Distribution of the moduli of the coefficients $\left|a_{1}\right|$ (short-dashed curve), $\left|a_{2}\right|$ (dotted), $\left|a_{4}\right|$ (dash-dotted curve) and $\left|a_{6}\right|$ (long-dashed curve) for CUE matrices of size $N=20$. Distributions of the two first coefficients are compared with theoretical predictions (narrow curves).

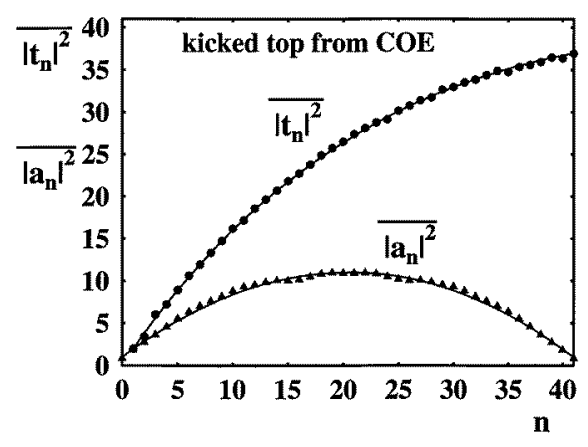

Figure 6. Mean squared traces $\overline{\left|t_{n}\right|^{2}}$ (circles) and coefficients $\overline{\left|a_{n}\right|^{2}}$ (triangles) for orthogonal tops are compared with COE results (curves). Data are averaged over 20000 different Floquet matrices of the structure (6.1) as described in the text.

were found as of the COE type. We shall refer to the two variants of the model as to the unitary and orthogonal top, respectively.

Before presenting our data for the traces $t_{n}$ and the secular coefficients $a_{n}$ of various tops a word of caution is in order. Previous statistical analyses of tops were made for spectrally local quantities such as low-order correlations of the level density, the distribution of nearestneighbour spacings, or for components of eigenvectors; what distinguishes these quantities is a certain self-averaging character. A single Floquet matrix of large dimension $N$ provides a sufficient data basis to extract reliable means or even distributions from. Now, the sequence of the traces $t_{n}$ and that of the secular coefficients $a_{n}$ are not in any way self-averaging since such a sequence with $1 \leqslant n \leqslant \frac{1}{2} N$ stands for a whole quasi-energy spectrum and therefore changes in a system specific manner when control parameters are varied. Consequently, it would not make sense to compare such a sequence for an individual Floquet matrix with the means calculated here for the various circular ensembles. We must rather ask whether an ensemble of Floquet matrices of the type (62), defined by a whole set of values for the various control parameters, is faithful to the prediction based on the circular ensemble of random matrices of the same symmetry class. It is in this sense that we have undertaken the comparison to follow.

Figure 6 shows the $n$ dependence of the variances of the traces (circles) and of the coefficients (triangles) for orthogonal tops. The order of the characteristic polynomial was taken as $N=41$ by choosing $j=20$. Data from 20000 matrices were gathered by

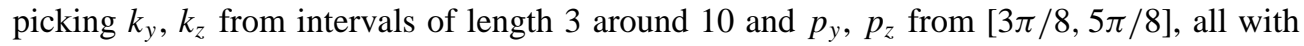
independent box distributions. These intervals were chosen so as to secure classical chaos and to avoid geometric symmetries. The agreement with the COE is obviously satisfactory.

Analogous data for unitary tops are presented in figure 7 . The agreement with the CUE predictions is acceptable in the sense that there is no doubt about the universality class. However, system specific behaviour is clearly visible for low-order traces and even 


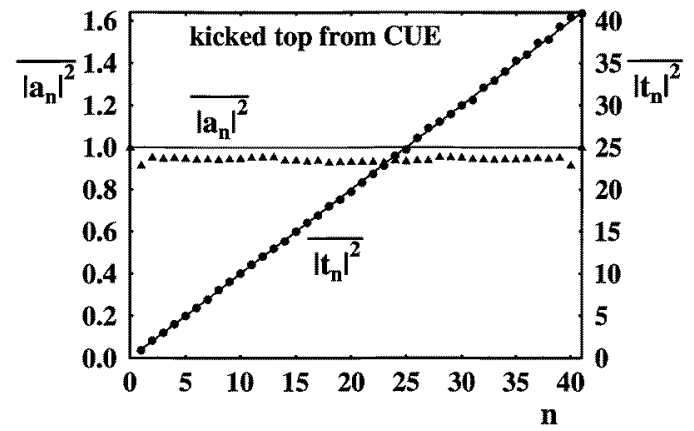

Figure 7. As in figure 6 for unitary tops. Curves denote the CUE results.

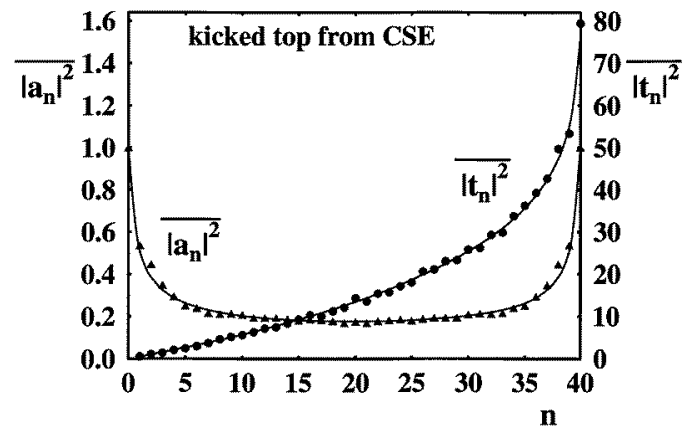

Figure 8. As in figure 6 for symplectic tops. Average over 2000 matrices as described in the text. Curves denote CSE results.

in all secular coefficients. Qualitatively, such deviations from random-matrix theory are not unexpected since low-order traces do contain primarily system specific information retrievable from short periodic orbits and since the $a_{n}$ even for large $n$ contain low-order traces as expressed in Newton's formulae.

Symplectic tops can also be constructed by securing an anti-unitary symmetry $T$ with $T^{2}=-1$ and avoiding geometric symmetries [3]. The Floquet operator

$$
\begin{gathered}
F=\exp \left(-\mathrm{i} \frac{k_{1}}{2 j+1} J_{z}^{2}-\mathrm{i} \frac{k_{2}}{2(2 j+1)}\left(J_{x} J_{z}+J_{z} J_{x}\right)-\mathrm{i} \frac{k_{3}}{2(2 j+1)}\left(J_{x} J_{y}+J_{y} J_{x}\right)\right) \\
\quad \times \exp \left(-\mathrm{i} \frac{k_{4}}{2 j+1} J_{z}^{2}\right)
\end{gathered}
$$

in a representation with half-integer $j$ is a good example. A set of 2000 matrices with $j=39.5$ and coupling constants $k_{i}$ drawn at random from a hypercube of length 0.4 near 10 gives data shown in figure 8 . The agreement with the CSE is better than could be hoped for.

\section{Acknowledgments}

We would like to thank Nils Lehmann for helpful cooperation and Pragya Shukla for first efforts to calculate the mean squared secular coefficients for the COE and the CSE. Financial support by the Sonderforschungsbereich 'Unordnung und große Fluktuationen' der Deutschen Forschungsgemeinschaft is gratefully acknowledged. 


\section{Appendix A. Differential equation for the generating function}

Let us recall the heuristic way we first approached the mean squared secular coefficients (19), (23), and (24). After deriving the Pfaffians (18) and (21) we applied an algebraic computer program to evaluate the $\overline{\left|a_{n}\right|^{2}}$ for small dimensions $N$ and then proceeded analytically to $n=1,2$ and arbitrary $N$. We thank $\mathrm{N}$ Lehmann for the computer work and also for his intuition in proposing (23) with us. Having eventually proposed (19) as well we speculatively extrapolated to the general form (24) for arbitrary positive $\beta$.

To prepare for the proof of (24) we observe the equivalence to the recursion relation

$$
(n+1)\left(1+\frac{\beta}{2}(N-n-1)\right) \overline{\left|a_{n+1}\right|^{2}}=(N-n)\left(1+n \frac{\beta}{2}\right) \overline{\left|a_{n}\right|^{2}}
$$

with $\overline{\left|a_{0}\right|^{2}}=1$. Multiplying (A1) by $x^{n}$, using $(x \partial / \partial x) x^{n}=n x^{n}$, and summing from $n=0$ to $n=N-1$ we obtain the differential equation (25), the polynomial solution of which with $P_{N}^{\beta}(x)=1$ generates the mean squared secular coefficients. A few elementary steps will now yield the validity of the differential equation.

We start from the definition (4) of the generating function, setting $\lambda=x, \mu=1$ and take the derivative with respect to $x$,

$$
\frac{\partial P(x)}{\partial x}=-\left\langle\sum_{i=1}^{N} \frac{1}{\mathrm{e}^{\mathrm{i} \varphi_{i}}-x}\right\rangle
$$

where the angular brackets denote an average with the whole integrand of (A2) as the weight. On the other hand, taking the derivative with respect to $\mu$ before setting $\mu=1$ and exploiting the invariance of the $N$-fold phase integral under a constant shift of all phases we get

$$
x \frac{\partial P(x)}{\partial x}=-\left\langle\sum_{i=1}^{N} \frac{1}{\mathrm{e}^{-\mathrm{i} \varphi_{i}}-1}\right\rangle .
$$

Next, we differentiate (A2) once more,

$$
\begin{aligned}
\frac{\partial^{2} P(x)}{\partial x^{2}} & =\left\langle\sum_{i \neq j} \frac{1}{\left(\mathrm{e}^{\mathrm{i} \varphi_{i}}-x\right)\left(\mathrm{e}^{\mathrm{i} \varphi_{j}}-x\right)}\right\rangle \\
& =\left\langle\sum_{i \neq j} \frac{-2}{\left(\mathrm{e}^{\mathrm{i} \varphi_{i}}-x\right)\left(\mathrm{e}^{\mathrm{i} \varphi_{i}}-\mathrm{e}^{\mathrm{i} \varphi_{j}}\right)}\right\rangle \\
& =-\left\langle\sum_{i \neq j} \frac{\mathrm{e}^{-\mathrm{i} \varphi_{i}}}{\left(\mathrm{e}^{\mathrm{i} \varphi_{i}}-x\right)}\left(1-\mathrm{i} \cot \frac{\varphi_{i}-\varphi_{j}}{2}\right)\right\rangle .
\end{aligned}
$$

Realizing that the cotangent function may be replaced by a derivative acting on the joint density of eigenvalues (2),

$$
\left(\frac{\partial}{\partial \varphi_{i}}-\frac{\beta}{2} \sum_{j(\neq i)} \cot \frac{\varphi_{i}-\varphi_{j}}{2}\right) d_{N}^{\beta}=0
$$

we can transform the last member in (A4) by partial integration. A little algebraic hocuspocus then gives, with the help of (A2) and (A3),

$$
\begin{aligned}
\frac{\partial^{2} P(x)}{\partial x^{2}}=(N & \left.-1+\frac{2(2-x)}{\beta(1-x)}\right) \frac{1}{x}\left(\frac{\partial P}{\partial x}+\sum_{i}\left\langle\mathrm{e}^{-\mathrm{i} \varphi_{i}}\right\rangle\right) \\
& +\frac{2}{\beta(1-x)}\left(-\sum_{i}\left\langle\mathrm{e}^{-\mathrm{i} \varphi_{i}}\right\rangle-N P+x \frac{\partial P}{\partial x}\right) .
\end{aligned}
$$


Finally, the average $\sum_{i}\left\langle\mathrm{e}^{-\mathrm{i} \varphi_{i}}\right\rangle$ can be expressed in terms of the generating function $P$ and its derivative $\partial P / \partial x$ by employing the identity $\sum_{k}\left\langle\mathrm{e}^{-\mathrm{i} \varphi_{k}}\right\rangle=\sum_{k}\left\langle\mathrm{i} \partial \mathrm{e}^{-\mathrm{i} \varphi_{k}} / \partial \varphi_{k}\right\rangle$, integrating by parts, and again invoking (A2), (A3) and (A5),

$$
\sum_{i}\left\langle\mathrm{e}^{-\mathrm{i} \varphi_{i}}\right\rangle\left(2+\frac{\beta}{2}(N-1)\right)=(x-1) \frac{\partial P}{\partial x}-N P .
$$

Upon inserting this in (A6) we obtain a differential equation for the generating function $P$ which is easily put into the form (25).

\section{References}

[1] Dyson F J 1962 J. Math. Phys. 3140

[2] Mehta M L 1991 Random Matrices II (New York: Academic)

[3] Haake F 1990 Quantum Signatures of Chaos (Berlin: Springer)

[4] Jalabert R A and Pichard J-L 1995 J. Physique I (France) 5287

[5] Gaudin M 1966 Nucl. Phys. 85545

[6] Pandey A and Shukla P 1991 J. Phys. A: Math. Gen. 243907

[7] Muttalib K A and Ismail M E H 1995 J. Phys. A: Math. Gen. 28 L541

[8] Lewenkopf C H and Weidenmüller H A 1991 Ann. Phys., NY 21253

[9] Brouwer P W 1995 Phys. Rev. B 5116878

[10] Pereyra P and Mello P A 1983 J. Phys. A: Math. Gen. 16237

[11] Mello P A, Pereyra P and Seligman T H 1985 Ann. Phys., NY 161254

[12] Doron E, Smilanski U and Frenkel A 1991 Physica 50D 367

[13] Baranger H U and Mello P A 1994 Phys. Rev. Lett. 73142

[14] Mostowski A and Stark M 1964 Introduction to Higher Algebra (Oxford: Pergamon)

[15] Bogomolny E, Bohigas O and Leboeuf P 1992 Phys. Rev. Lett. 682726

[16] Kuś M, Haake F and Eckhardt B 1993 Z. Phys. B 92221

[17] Bogomolny E, Bohigas O and Leboeuf P 1995 Preprint

[18] Życzkowski K and Kuś M 1994 J. Phys. A: Math. Gen. 274235

[19] Życzkowski K 1995 Chaos-the interplay between stochastic and deterministic behavior Proc. XXXIst Winter School of Theoretical Physics (Karpacz, 1995) ed P Garbaczewski, M Wolf and A Weron (Berlin: Springer) p 565

[20] Życzkowski K and Kuś M 1996 Phys. Rev. E 53319

[21] Abramowitz M and Stegun I A (eds) 1970 Handbook of Mathematical Functions (New York: Dover)

[22] Hartwig R E and Fisher M E 1969 Arch. Rat. Mech. Anal. 32190

[23] Haake F, Kuś M and Scharf R 1987 Z. Phys. B 65381 